\title{
Erratum: The shape of a memorised random walk
}

Michał Gnacik, Abdulrahman Alsolami and James Burridge

School of Mathematics and Physics, University of Portsmouth, Portsmouth,

PO1 3HF, United Kingdom

E-mail: james.burridge@port.ac.uk and michal.gnacik@port.ac.uk

An error was introduced during the typesetting of this manuscript, " $c \rightarrow \infty$ is" is missing from the manuscript. Theorem 1 should read:

Theorem 1 (Egocentric Asphericity). The egocentric asphericity of $2 D$ Brownian motion memorised according to $\mu$, that is, $(X(t), Y(t))_{t \in S}$, as $c \rightarrow \infty$ is

$$
A_{2}=1-4 \lim _{\tau \rightarrow \infty} \frac{\alpha(\tau)}{\beta(\tau)},
$$

where

$$
\begin{aligned}
\alpha(\tau)= & \frac{1}{2}\left[\left(\int_{0}^{\tau} s \mu(s) \mathrm{d} s\right)^{2}+\left(\int_{0}^{\tau} M(s) \mathrm{d} s\right)^{2}-\tau^{2} M^{2}(\tau)\right] \\
& +\int_{0}^{\tau}[(4 s-\tau) M(\tau)-2 s M(s)] M(s) \mathrm{d} s \\
\beta(\tau)=2 & {\left[\left(\int_{0}^{\tau} s \mu(s) \mathrm{d} s\right)^{2}+\left(\int_{0}^{\tau} M(s) \mathrm{d} s\right)^{2}+3 \tau^{2} M^{2}(\tau)\right] } \\
& -4 \int_{0}^{\tau}[(4 s+\tau) M(\tau)-2 s M(s)] M(s) \mathrm{d} s .
\end{aligned}
$$

